\title{
A Uniqueness Theorem for Anticommutation Relations and Commutation Relations of Quantum Spin Systems
}

\author{
P. Combe ${ }^{1}$, R. Rodriguez ${ }^{1}$, M. Sirugue-Collin ${ }^{2}$, and M. Sirugue ${ }^{3}$ \\ ${ }^{1}$ Université d'Aix-Marseille II, U.E.R. Scientifique de Luminy, and \\ Centre de Physique Théorique, C.N.R.S., F-13274 Marseille Cedex 2, France \\ ${ }^{2}$ Université de Provence and Centre de Physique Théorique, C.N.R.S., \\ F-13274 Marseille Cedex 2, France \\ ${ }^{3}$ Centre de Physique Théorique, C.N.R.S., F-13274 Marseille Cedex 2, France
}

\begin{abstract}
Under quite natural assumptions we prove that a classical spin lattice has essentially two natural quantum extensions: The quantum spin lattice and the Fermi system. Moreover we derive a transformation from a commuting pair of Fermi systems to a purely anticommuting Fermi system.
\end{abstract}

\section{Introduction}

It is known that quantum spin commutation relations and anticommutation relations can be extracted from the central extension of an abelian group, in a quite similar manner as usual commutation relations can be extracted from central extension of an abelian group, namely the abelian group of the phase space. (See e.g. $[1,2]$ ); this point of view has obvious advantages not only aesthetical, and we postpone to a forthcoming paper some results which are transcribed from the case of quantum canonical commutation relations. Our aim in this paper is to derive a result which is analogous to the fact that, up to the value of $\hbar$ (the Planck constant), there is essentially one possible central extension of the group $\mathbb{R}^{2}$. In our case for a given value of $\hbar$ there are essentially four possible central extensions of $\mathfrak{p}_{A} \times \mathfrak{p}_{A}$ [see Definition (2.1)] satisfying some homogeneity requirements. Two of them are rather trivial from a physical point of view and the two others correspond to the usual quantum spin systems and Fermi systems.

Nevertheless the $C^{*}$-algebras which are built in a canonical way on these extensions are unique up to isomorphisms if the number of points in $\Lambda$ is even or infinite.

The $*$-isomorphisms between the two algebras are of especially simple form, namely:

$$
\hat{\alpha}\left(\delta_{X, Y}^{\prime}\right)=f(X, Y) \delta_{\tau(X, Y)},
$$

where $f$ is a function from $\mathfrak{p}_{\Lambda} \times \mathfrak{p}_{\Lambda}$ to \pm 1 and $\tau$ an isomorphism of $\mathfrak{p}_{A} \times \mathfrak{p}_{A}$ onto itself [Theorem (2.40)].

This theorem contains an abstract version of an old trick which connects the Clifford algebra to the U.H.F. algebra (see e.g. $[3,4]$ ). But it contains also as 
corollary [Corollary $(2.41)]$ the following result: if $\mathscr{H}_{1}$ and $\mathscr{H}_{2}$ are real Hilbert space of even or infinite dimension $\mathscr{H}_{1} \oplus \mathscr{H}_{2}$ their orthogonal sum then:

$$
\mathfrak{U}\left(\mathscr{H}_{1} \oplus \mathscr{H}_{2}\right) \simeq \mathfrak{U}\left(\mathscr{H}_{1}\right) \otimes \mathfrak{U}\left(\mathscr{H}_{2}\right),
$$

where $\mathfrak{A}\left(\mathscr{H}_{i}\right), \mathfrak{A}(\mathscr{H})$ are the Clifford algebras built on $\mathscr{H}_{i}, \mathscr{H}$ and the $*$-isomorphisms can be chosen to send a finite product of generators in the left hand side onto a finite product of generators in the right hand side.

\section{Central Extension of Abelian Groups by the Torus}

This section contains results which are needed for Sect. 2; some of them are known, if not in the full generality that we shall need in the sequel. We shall always consider groups equipped with the discrete topology.

Definition (1.1.). Let $G$ be an abelian group. A multiplier on $G$ is a function $\xi: G \times G \rightarrow T$, which satisfies:

$$
\xi\left(g_{1}, g_{2}\right) \xi\left(g_{1} g_{2}, g_{3}\right)=\xi\left(g_{2}, g_{3}\right) \xi\left(g_{1}, g_{2} g_{3}\right), \quad \forall g_{1}, g_{2}, g_{3} \in G
$$

The next proposition is an easy consequence of the previous definition:

Proposition (1.2). Let $G$ be an abelian group, $\xi$ a multiplier on $G$, then:

$$
\xi(e, g)=\xi(g, e)=\xi(e, e), \quad \xi\left(g, g^{-1}\right)=\xi\left(g^{-1}, g\right), \quad \forall g \in G .
$$

The usefulness of the multipliers is examplified in the following

Proposition (1.3). Let $G$ be an abelian group, $\xi$ a multiplier on $G$, then $G \times T$ endowed with the product:

$$
(g, \alpha)\left(g^{\prime}, \alpha^{\prime}\right)=\left(g g^{\prime}, \xi\left(g, g^{\prime}\right) \alpha \alpha^{\prime}\right), \quad \forall g, g^{\prime} \in G, \quad \alpha, \alpha^{\prime} \in T
$$

and the inverse law:

$$
(g, \alpha)^{-1}=\left(g^{-1}, \overline{\xi(e, e)} \overline{\xi\left(g, g^{-1}\right)} \bar{\alpha}\right), \quad \forall g \in G, \alpha \in T
$$

is a group $G \otimes_{\xi} T$, with identity $(e, \overline{\xi(e, e)})$, the central extension of $G$ by $T$. Namely one has the following exact sequence

$$
e \rightarrow T \rightarrow G \otimes_{\xi} T \rightarrow G \rightarrow e .
$$

Multipliers on $G$ form an abelian group for pointwise multiplication, and the subgroup of trivial multipliers of the form

$$
\zeta\left(g, g^{\prime}\right)=\lambda(g) \lambda\left(g^{\prime}\right) \overline{\lambda\left(g g^{\prime}\right)}, \quad \forall g, g^{\prime} \in G,
$$

where $\lambda$ is any function from $G \rightarrow T$ form an invariant subgroup. The quotient of these two groups characterizes the different extensions up to isomorphism, in the following sense.

Proposition (1.4). Let $G$ be an abelian group; $\xi$ and $\zeta$ two multipliers. The necessary and sufficient condition for the existence of an isomorphism $j$ between $G \otimes_{\xi} T$ and 
$G \otimes_{\zeta} T$ which makes the following diagram commutative:<smiles>C=C[Te][Te]=[Te]</smiles>

is that there exists a function $\lambda$ from $G$ to $T$ such that:

$$
\xi\left(g, g^{\prime}\right)=\lambda(g) \lambda\left(g^{\prime}\right) \overline{\lambda\left(g g^{\prime}\right)} \zeta\left(g, g^{\prime}\right), \quad \forall g, g^{\prime} \in G
$$

We can use this fact to simplify the calculations, so:

Proposition (1.5). Let $G$ be an abelian group, $\xi$ a multiplier on $G$. There exists a multiplier $\zeta$ which is equal to $\xi$ up to a trivial multiplier, such that:

$$
\zeta(e, e)=\zeta\left(g, g^{-1}\right)=1 \text {. }
$$

We can give explicitly the isomorphism $j$ between the two groups $G \otimes_{\xi} T$ and $G \otimes \otimes_{\zeta} T:$

$$
j\left((g, \alpha)_{G \otimes_{\zeta} T}\right)=(g, \overline{\lambda(g)} \alpha)_{G \otimes_{\xi} T}, \quad \forall g \in G, \alpha \in T,
$$

where the subscripts indicate in which groups we consider the elements $(g, \alpha)$ since as sets the two groups are equal.

In the case of groups whose elements are of order 2, according to a theorem of Kleppner (see [5]), every multiplier is equivalent to a bicharacter. In [6] the case where $\xi$ is a bicharacter has been studied explicitly but most of the results are still valid without this assumption [see Proposition (1.12)].

For classifying the different central extensions of a group $G$ [see Theorem (2.24)] it is more convenient to deal with the bicharacter which is associated with a multiplier since it has more special properties; hence it can be characterized in a easier way. Indeed if $G$ is an abelian group and $\xi$ a multiplier, $b_{\xi}$ :

$$
b_{\xi}\left(g, g^{\prime}\right)=\xi\left(g, g^{\prime}\right) \overline{\xi\left(g^{\prime}, g\right)}, \quad \forall g, g^{\prime} \in G,
$$

is a bicharacter of $G$. It characterizes uniquely the extension according to the proposition:

Proposition (1.8). Let $G$ be an abelian group, $\xi$ and $\zeta$ two multipliers, the necessary and sufficient condition for $\xi$ and $\zeta$ to be equal up to a trivial multiplier is that:

$$
b_{\xi}=b_{\zeta} .
$$

Bicharacters associated with multipliers satisfy special properties:

$$
\begin{aligned}
& b_{\xi}(g, g)=1, \\
& b_{\xi}\left(g, g^{\prime}\right)=\widetilde{b_{\xi}\left(g^{\prime}, g\right)} .
\end{aligned}
$$

In the case of groups whose elements are of order 2 , the antisymmetry relation (1.9) becomes a symmetry relation, namely:

$$
b_{\xi}\left(g, g^{\prime}\right)=b_{\xi}\left(g^{\prime}, g\right), \quad \forall g, g^{\prime} \in G \quad \text { such that } \quad g^{2}=g^{\prime 2}=e .
$$


Moreover we shall say that a multiplier $\xi$ is non degenerate if

$$
\left\{g \in G ; b_{\xi}\left(g, g^{\prime}\right)=1, \forall g^{\prime} \in G\right\}=\{e\} .
$$

In [6] we gave an explicit construction of a $C^{*}$-algebra whose representations are in bijection with the unitary representations of $G \otimes_{\xi} T$ where the center of $G \otimes_{\xi} T$ is trivially represented, for $\xi$ a bicharacter. Actually all we did there can be repeated for $\xi$ a multiplier, namely:

Proposition (1.12). Let $G$ be an abelian group and $\xi$ a non degenerate multiplier. Let $\Delta(G, \xi)$ be the set of functions from $G$ to $\mathbb{C}$ with finite support, equipped with the following product:

$$
F_{*} G(g)=\sum_{g^{\prime} \in G} \xi\left(g g^{\prime-1}, g^{\prime}\right) F\left(g g^{\prime-1}\right) G\left(g^{\prime}\right)
$$

and *-operation:

$$
F^{*}(g)=\overline{\xi(e, e)} \overline{\xi\left(g, g^{-1}\right)} \overline{F\left(g^{-1}\right)} .
$$

$\Delta(G, \xi)$ is a *-algebra which has a unique norm of $C^{*}$-algebra. Let $\overline{\Delta(G, \xi)}$ its closure. The *-representations of $\overline{\Delta(G, \xi)}$ are in bijection with the unitary representations $U$ of $G \otimes_{\xi} T$ for which

$$
U(g, \alpha)=\alpha U(g, 1), \quad \forall g \in G, \alpha \in T .
$$

The basic generators of $\overline{\Delta(G, \xi)}$ are the functions $\delta_{g}, g \in G$, such that

$$
\delta_{g}\left(g^{\prime}\right)= \begin{cases}1 & \text { if } g=g^{\prime} \\ 0 & \text { otherwise }\end{cases}
$$

which of course satisfy:

$$
\begin{aligned}
& \delta_{g^{*}} * \delta_{g^{\prime}}=\xi\left(g, g^{\prime}\right) \delta_{g g^{\prime}}, \quad \forall g, g^{\prime} \in G, \\
& \delta_{g}^{*}=\overline{\xi(e, e)} \overline{\xi\left(g, g^{-1}\right)} \delta_{g^{-1}}
\end{aligned}
$$

the algebra $\overline{\Delta(G, \xi)}$ has interesting properties with respect to automorphisms, namely:

Proposition (1.16). Let $G$ be an abelian group. Let $\xi$ and $\zeta$ be multipliers on $G$; let $\alpha$ be an isomorphism of $G$ onto $G$ such that there exists a function $\lambda: G \rightarrow T$ with the property:

$$
\xi\left(\alpha(g), \alpha\left(g^{\prime}\right)\right)=\lambda(g) \lambda\left(g^{\prime}\right) \overline{\lambda\left(g g^{\prime}\right)} \zeta\left(g, g^{\prime}\right)
$$

then there exists a $*$-isomorphism $\hat{\alpha}$ of $\overline{\Delta(G, \zeta)}$ onto $\overline{\Delta(G, \xi)}$ :

$$
\hat{\alpha}\left(\delta_{g}^{\zeta}\right)=\overline{\lambda(g)} \delta_{\alpha(g)}^{\zeta}, \quad \forall g \in G .
$$

In particular $\alpha$ can be the identity isomorphism which shows that $\overline{\Delta(G, \xi)}$ depends on $\xi$ up to a trivial multiplier.

It is easy to prove that $\alpha$ is a $*$-isomorphism of $\Delta(G, \zeta)$ onto $\Delta(G, \xi)$. Then one uses an obvious extension of Theorem (3.10) of [6]. 
Finally let us remark that the bicharacter $b_{\xi}$ associated to a multiplier is directly associated to the commutation relations, indeed:

Proposition (1.17). Let $G$ be an abelian group and $\xi$ a multiplier, then:

$$
\delta_{g^{\prime}} * \delta_{g^{\prime}}=b_{\xi}\left(g, g^{\prime}\right) \delta_{g^{\prime}} * \delta_{g}, \quad \forall g \cdot g^{\prime} \in G
$$

so that the non degeneracy of $\xi$ implies that $\overline{\Delta(G, \xi)}$ has no non trivial center, see also [6].

In the next section we shall specialize ourselves to a special group connected with both quantum spin commutation relations and anticommutation relations. We shall show that the central extensions of this group are essentially unique.

\section{The Basic Phase Space Group for Spin Systems and Anticommutation Relations}

It is possible to obtain the commutation relations of quantum spin systems and anticommutation relations from the central extension of a group that we shall describe in the following.

Definition (2.1). Let $A$ be at most a countable set; $\mathfrak{p}_{A}$ (resp. $\mathscr{P}_{A}$ ) is the group of finite subsets (resp. all subsets) of $\Lambda$ equipped with the symmetric difference as a group law: viz.

$$
\forall X_{1}, X_{2}, \ldots, X_{n} \in \mathscr{P}_{\Lambda}, \quad X_{1} \triangle X_{2} \triangle \ldots \triangle X_{n}
$$

is the subset of $\Lambda$ whose points belong at most to an odd number of $X_{i}$ 's.

This law is clearly associative and symmetric; the identity element is the void subset:

(2.3) $X \triangle \emptyset=X, \quad \forall X \subset A$,

and every element is of order 2 :

(2.4) $X \triangle X=\emptyset, \quad \forall X \subset \Lambda$.

$\mathfrak{p}_{A}$ is a subset of $\mathscr{P}_{A}$; they coincide if $\Lambda$ is finite. $\mathfrak{p}_{A}$ and $\mathscr{P}_{A}$ have natural topologies, namely for $\mathfrak{p}_{A}$ the discrete topology; $\mathscr{P}_{A}$ is isomorphic to the topological product of $\{0,1\}^{A}$, hence compact for the product topology and it is isomorphic to the dual group of $p_{A}$, through the natural bilinear form:

$$
(X, Y) \in \mathfrak{p}_{A} \times \mathscr{P}_{A} \rightarrow(X \mid Y)=(-1)^{|X \cap Y|},
$$

where $|X|$ denotes the number of points in $X$.

The structure of automorphisms of $p_{A}$ is especially simple.

Lemma (2.6). Let $\tau$ be a homomorphism of $p_{\Lambda}$, it is completely characterized by a function

$$
x_{i} \in \Lambda \rightarrow \tau\left(\left\{x_{l}\right\}\right) \in \mathfrak{p}_{A},
$$

so

$$
\tau(X)=\tau\left(\triangle_{x_{1} \in X}^{\triangle}\left\{x_{i}\right\}\right)=\triangle_{x_{i} \in X} \tau\left(\left\{x_{i}\right\}\right) .
$$


Actually for describing the commutation relations of a quantum spin system or anticommutation relations, we need the group $\mathfrak{p}_{\Lambda} \times \mathfrak{p}_{\Lambda}$. Despite of the fact that it is isomorphic to some $p_{\Lambda^{\prime}}$ we want to quote some more special results:

Proposition (2.7). Any isomorphism $\tau$ of $\mathfrak{p}_{A} \times \mathfrak{p}_{A}$ into itself is of the form

$$
\tau(X, Y)=\left(X \triangle F_{1}(X) \triangle F_{2}(Y), Y \triangle G_{1}(Y) \triangle G_{2}(X)\right),
$$

where the $F_{i}$ 's and $G_{i}$ 's are homomorphisms of $\mathfrak{p}_{A}$ such that the two equations

$$
X=F_{1}(X) \triangle F_{2}(Y), \quad Y=G_{1}(Y) \triangle G_{2}(X),
$$

have the unique solution $X=Y=\emptyset$; moreover if $\tau$ commutes with $\tau_{\theta_{0}}$ :

$$
\tau_{\theta_{0}}(X, Y)=(Y, X), \quad \forall X, Y \in \mathfrak{p}_{\Lambda},
$$

then

$$
F_{1}=G_{1} \text { and } F_{2}=G_{2} .
$$

The proof is obvious; notice that the $F_{i}$ 's are not necessarily isomorphisms as examplified by the following.

Proposition (2.8). Let $\theta$ be a homomorphism of $\mathfrak{p}_{A}$ into itself. Then:

$$
\tau_{\theta}(X, Y)=(X \triangle \theta(X \triangle Y), Y \triangle \theta(X \triangle Y)), \quad \forall X, Y \in \mathfrak{p}_{\Lambda},
$$

is an isomorphism of $p_{A} \times p_{A}$ onto itself. Moreover $\tau$ is an isomorphism of the group of homomorphisms of $\mathfrak{p}_{A}$ into itself onto the group of isomorphisms of $\mathfrak{p}_{A} \times \mathfrak{p}_{A}$ onto itself, which commutes with $\tau_{\theta_{0}}$ and preserves pointwise the diagonal $\Delta=\{(X, X)$; $\left.X \in \mathfrak{p}_{A}\right\}$; moreover $\tau_{\theta}^{2}=i$ the identity isomorphism.

Proof. If an isomorphism $\tau$ commutes with $\tau_{\theta_{0}}$ and preserves pointwise the diagonal $\Delta$ then:

$$
F_{1}(X) \triangle F_{2}(X)=\emptyset, \quad \forall X \in \mathfrak{p}_{\Lambda} .
$$

so that $F_{1}=F_{2}$ and $\tau$ rewrites:

$$
\tau(X, Y)=\left(X \triangle F_{1}(X \triangle Y), Y \triangle F_{1}(X \triangle Y)\right) .
$$

Conversely let $\theta$ be a homomorphism of $\mathfrak{p}_{A} ; \tau_{\theta}$ is injective since the equations

$$
\begin{aligned}
& X=\theta(X \triangle Y) \\
& Y=\theta(X \triangle Y)
\end{aligned}
$$

imply $X \triangle Y=\emptyset$ and $\theta(X \triangle Y)=\emptyset$; hence $X=Y=\emptyset$. It is surjective since its square is the identity isomorphism:

$$
\begin{aligned}
& \begin{aligned}
\tau_{\theta}^{2}(X, Y) & =\tau_{\theta}(X \triangle \theta(X \triangle Y), Y \triangle \theta(X \triangle Y)) \\
& =(X \triangle \theta(X \triangle Y) \triangle \theta(X \triangle \theta(X \triangle Y) \triangle Y \triangle \theta(X \triangle Y))
\end{aligned} \\
& Y \triangle \theta(X \triangle Y) \triangle \theta(X \triangle \theta(X \triangle Y) \triangle Y \triangle \theta(X \triangle Y))=(X, Y), \quad \forall X, Y \in \mathfrak{P}_{\Lambda} .
\end{aligned}
$$

This relation is a special case of the obvious relation:

$$
\tau_{\theta_{1}} \circ \tau_{\theta_{2}}=\tau_{\theta_{1} \Delta \theta_{2}}
$$


where $\theta_{1} \Delta \theta_{2}$ is defined for a pair of homomorphisms of $\mathfrak{p}_{A}$ into itself by:

$$
\theta_{1} \triangle \theta_{2}(X)=\theta_{1}(X) \triangle \theta_{2}(X), \quad \forall X \in \mathfrak{p}_{\Lambda} ;
$$

they form a group with identity:

$$
\theta_{\emptyset}(X)=\emptyset, \quad \forall X \in \mathfrak{p}_{\Lambda} ;
$$

another remarkable homomorphism is $\theta_{0}$ :

(2.13) $\theta_{0}(X)=X, \quad \forall X \in \mathfrak{p}_{\Lambda}$.

Among these isomorphisms we shall need two special ones which are described in the following definition.

Definition (2.14). $\tau_{1}=\tau_{\theta_{1}}$ and $\tau_{2}=\tau_{\theta_{2}}$ are the isomorphisms of $\mathfrak{p}_{A} \times \mathfrak{p}_{A}$ which come from the homomorphisms $\theta_{1}$ and $\theta_{2}$ of $p_{A}$ which are defined by

$$
\begin{aligned}
\theta_{1}\left(\left\{x_{1}\right\}\right) & =\emptyset, \\
\theta_{1}\left(\left\{x_{i}\right\}\right) & =\left\{x_{j} \in \Lambda ; j<i\right\}, \quad \forall i>1 . \\
\theta_{2}\left(\left\{x_{1}\right\}\right) & =\emptyset, \\
\theta_{2}\left(\left\{x_{2 k+1}\right\}\right) & =\left\{x_{j} \in \Lambda ; j<2 k+1\right\}, \quad \forall k>0, \\
\theta_{2}\left(\left\{x_{2 k}\right\}\right) & =\left\{x_{j} \in \Lambda ; j \leqq 2 k\right\}, \quad \forall k \geqq 1,
\end{aligned}
$$

for an arbitrary given order on the points in $\Lambda$.

We give in the next proposition some formulas which are useful in the following.

Proposition (2.15). Let $\theta_{i}$ be the homomorphisms defined in the previous proposition then one has the relations:

$$
\begin{aligned}
& \left|X \cap \theta_{1}(Y)\right|+\left|Y \cap \theta_{1}(X)\right| \\
& =|X||Y|+|X \cap Y| \bmod 2 \quad \forall X, Y \in \mathfrak{p}_{A},
\end{aligned}
$$

(2.17) $\left|X \cap \theta_{1}(X)\right|=\frac{1}{2}|X|(|X|-1)+|X|(|X|-1)(|X|-2) \quad \bmod 4 \quad \forall X \in \mathfrak{p}_{\Lambda}$,

(2.18) $\left|\theta_{2}(X)\right|=0 \bmod 2 \quad \forall X \in \mathfrak{p}_{\Lambda}$,

(2.19) $\left|X \cap \theta_{2}(Y)\right|+\left|Y \cap \theta_{2}(X)\right|=|X||Y|+|X \cap Y| \quad \bmod 2 \quad \forall X, Y \in \mathfrak{p}_{\Lambda}$,

(2.20) $\left|X \cap \theta_{2}(X)\right|-\left|X \cap \theta_{1}(X)\right|=\left|\theta_{2}(X)\right|-\left|\theta_{1}(X)\right| \quad \forall X \in \mathfrak{p}_{A}$.

We shall not prove these relations since the calculations are easy but tedious.

Let now $\sigma \in \Xi$ be a finite permutation of the points in $\Lambda$.

$$
\theta_{\sigma}\left(\left\{x_{i}\right\}\right)=\left\{x_{i}\right\} \triangle\left\{x_{\sigma(i)}\right\} \quad \forall x_{i} \in \Lambda
$$

induces an isomorphism $\tau_{\sigma}$ of $\mathfrak{p}_{A} \times \mathfrak{p}_{A}$ which commutes with $\tau_{\theta_{0}}$ and represents the finite permutations by taking in Proposition (2.7)

$$
\begin{aligned}
& F_{1}(X)=\theta_{\sigma}(X), \quad \forall X \in \mathfrak{p}_{\Lambda}, \\
& F_{2}(X)=\theta_{\emptyset}(X)=\emptyset, \quad \forall X \in \mathfrak{p}_{\Lambda} .
\end{aligned}
$$


This isomorphism does not preserve pointwise the diagonal; it is an isomorphism since $X \triangle \theta_{\sigma}(X)=\emptyset$ implies that $X=\theta_{\sigma}(X)$ but

$$
\theta_{\sigma}(X)=\underset{x_{i} \in X}{\triangle} \theta_{\sigma}\left(\left\{x_{i}\right\}\right)=\triangle \triangle_{x_{i} \in X}\left\{x_{i}\right\} \triangle\left\{x_{\sigma(i)}\right\},=X \triangle \sigma(X)
$$

where $\sigma(X)=\left\{x_{\sigma(i)} ; x_{i} \in X\right\}$,

hence $\sigma(X)=\emptyset$ and $X=\emptyset$.

This group of isomorphisms is of central importance to reduce the number of possible central extensions of $\mathfrak{p}_{A} \times \mathfrak{p}_{A}$ which is our next aim.

As we noticed any central extension of $\mathfrak{p}_{\Lambda} \times \mathfrak{p}_{\Lambda}$ by the torus is completely characterized up to the equivalence by a bicharacter $b_{\xi}$ which is both symmetric and satisfies:

$$
b_{\xi}((X, Y),(X, Y))=1
$$

using this remark we can state the

Theorem (2.24). Let $\mathrm{p}_{\Lambda}$ be the group of finite subsets of a set $\Lambda(|\Lambda|=2 N$ or $\infty)$ endowed with the symmetric difference as group operation; let $\mathfrak{p}_{\Lambda} \times \mathfrak{p}_{\Lambda}$ be the direct product of $\mathfrak{p}_{\Lambda}$ by itself. Let $\mathbb{S}$ be the group of the finite permutations of $\Lambda$; denote by $\tau_{\sigma}$ the induced isomorphism of $\mathfrak{p}_{\Lambda} \times p_{\Lambda}$ ( see (2.21)).

$$
\tau_{\sigma}(X, Y)=\left(X \triangle \theta_{\sigma}(X), Y \triangle \theta_{\sigma}(Y)\right), \quad \forall X, Y \in \mathfrak{p}_{\Lambda} .
$$

Denote by $\tau_{\theta_{0}}$ the automorphism of $\mathfrak{p}_{A} \times \mathfrak{p}_{A}$ :

$$
\tau_{\theta_{0}}(X, Y)=(Y, X), \quad \forall X, Y \in \mathfrak{p}_{\Lambda}
$$

then there exists only four bicharacters $b$ on $\mathfrak{p}_{\Lambda} \times \mathfrak{p}_{\Lambda}$ which are

i) symmetric, i.e. $b\left((X, Y),\left(X^{\prime}, Y^{\prime}\right)\right)=b\left(\left(X^{\prime}, Y^{\prime}\right),(X, Y)\right)$

ii) invariant by $\tau_{\sigma}$ for any $\sigma \in \mathcal{S}$, viz

$$
b\left(\tau_{\sigma}(X, Y), \tau_{\sigma}\left(X^{\prime}, Y^{\prime}\right)\right)=b\left((X, Y),\left(X^{\prime}, Y^{\prime}\right)\right)
$$

iii) invariant by $\tau_{\theta_{0}}$, viz

$$
b\left(\tau_{\theta_{0}}(X, Y), \tau_{\theta_{0}}\left(X^{\prime}, Y^{\prime}\right)\right)=b\left((X, Y),\left(X^{\prime}, Y^{\prime}\right)\right)
$$

iv) non degenerated, viz

$$
b\left((X, Y),\left(X^{\prime}, Y^{\prime}\right)\right)=1, \quad \forall X^{\prime}, Y^{\prime} \in \mathfrak{p}_{\Lambda} \Rightarrow X=Y=\emptyset .
$$

v) and satisfy:

$$
b((X, Y),(X, Y))=1, \quad \forall X, Y \in \mathfrak{p}_{\Lambda} .
$$

They are respectively:

$$
\begin{aligned}
& b^{S}\left((X, Y),\left(X^{\prime}, Y^{\prime}\right)\right)=(-1)^{\left|X \cap Y^{\prime}\right|+\left|X^{\prime} \cap Y\right|} \\
& b^{F}\left((X, Y),\left(X^{\prime}, Y^{\prime}\right)\right)=(-1)^{\left|X \cap X^{\prime}\right|+\left|Y \cap Y^{\prime}\right|+(|X|+|Y|)\left(\left|X^{\prime}\right|+\left|Y^{\prime}\right|\right)} \\
& b^{\varphi}\left((X, Y),\left(X^{\prime}, Y^{\prime}\right)\right)=(-1)^{\left|X \cap X^{\prime}\right|+\left|Y \cap Y^{\prime}\right|+|X|\left|X^{\prime}\right|+|Y|\left|Y^{\prime}\right|} \\
& b^{\sigma}\left((X, Y),\left(X^{\prime}, Y^{\prime}\right)\right)=(-1)^{\left|X \cap Y^{\prime}\right|+\left|X^{\prime} \cap Y\right|+|X|\left|Y^{\prime}\right|+\left|X^{\prime}\right||Y|}
\end{aligned}
$$


The two bicharacters $b^{\sigma}$ and $b^{\varphi}$ are degenerate when $|\Lambda|=2 k+1$. In what follows we denote $b\left((X, Y) ;\left(X^{\prime}, Y^{\prime}\right)\right)$ by $b\left(X, Y ; X^{\prime}, Y^{\prime}\right)$. Proof of theorem (2.24) uses a number of lemmas.

Lemma (2.25). Let $\chi$ be a bicharacter of $\mathfrak{p}_{A}$ which is invariant by $\sigma$ that is:

$$
\chi(\sigma(X), \sigma(Y))=\chi(X, Y) \quad \forall X, Y \in \mathfrak{p}_{\Lambda}, \sigma \in \Theta
$$

then it is of the form:

$$
\chi(X, Y)=(-1)^{\alpha|X \cap Y|+\beta|X||Y|}
$$

where $\alpha$ and $\beta$ are 0 or 1 .

Indeed if $X \neq \emptyset, Y \neq \emptyset$ then:

$$
\chi(X, Y)=\prod_{i \in X} \prod_{j \in Y} \chi(\{i\},\{j\}) .
$$

Since $\chi$ is permutation invariant, $\chi(\{i\},\{i\})$ is independent of $i$, the same for $\chi(\{i\},\{j\})(i \neq j)$ since if $i \neq j$ and $k \neq l$ there is a finite permutation $\sigma$ such that $\sigma(i)=k, \sigma(j)=l$; moreover $\chi(\{i\},\{j\})= \pm 1$; hence the four possibilities:

i) $\chi(\{i\},\{i\})=\chi(\{i\},\{j\})=1, \chi(X, Y)=1, \alpha=\beta=0$.

ii) $\chi(\{i\},\{i\})=-1, \chi(\{i\},\{j\})=1, \chi(X, Y)=(-1)^{|X \cap Y|}, \alpha=1, \beta=0$.

iii) $\chi(\{i\},\{i\})=1, \chi(\{i\},\{j\})=-1, \chi(X, Y)=(-1)^{|X||Y|+|X \cap Y|}, \alpha=\beta=1$.

iv) $\chi(\{i\},\{i\})=-1, \chi(\{i\},\{j\})=-1, \chi(X, Y)=(-1)^{|X||Y|}, \alpha=0, \beta=1$.

Notice that they are automatically symmetric

$$
\chi(X, Y)=\chi(Y, X) \text {. }
$$

Lemma (2.26). Any symmetric, $\subseteq$ invariant, $\tau_{\theta_{0}}$ invariant, bicharacter of $\mathfrak{p}_{\Lambda} \times \mathfrak{p}_{\Lambda}$ is of the form:

$$
\begin{aligned}
& b\left(X, Y ; X^{\prime}, Y^{\prime}\right) \\
& =(-1)^{\alpha_{1}\left(\left|X \cap X^{\prime}\right|+\left|Y \cap Y^{\prime}\right|\right)+\alpha_{2}\left(\left|X \cap Y^{\prime}\right|+\left|Y \cap X^{\prime}\right|\right)}(-1)^{\beta_{1}\left(|X|\left|X^{\prime}\right|+|Y|\left|Y^{\prime}\right|\right)+\beta_{2}\left(|X|\left|Y^{\prime}\right|+\left|X^{\prime}\right||Y|\right)} .
\end{aligned}
$$

Actually since $b$ is a bicharacter

$$
b\left(X, Y ; X^{\prime}, Y^{\prime}\right)=b\left(X, \emptyset ; X^{\prime}, \emptyset\right) \cdot b\left(X, \emptyset ; \emptyset, Y^{\prime}\right) \cdot b\left(\emptyset, Y ; X^{\prime}, \emptyset\right) \cdot b\left(\emptyset, Y ; \emptyset, Y^{\prime}\right) \text {. }
$$

but

$$
\begin{aligned}
& \chi_{1}\left(X, X^{\prime}\right)=b\left(X, \emptyset ; X^{\prime}, \emptyset\right), \\
& \chi_{2}\left(X, Y^{\prime}\right)=b\left(X, \emptyset ; \emptyset, Y^{\prime}\right), \\
& \chi_{3}\left(Y, X^{\prime}\right)=b\left(\emptyset, Y ; X^{\prime}, \emptyset\right), \\
& \chi_{4}\left(Y, Y^{\prime}\right)=b\left(\emptyset, Y ; \emptyset, Y^{\prime}\right) .
\end{aligned}
$$

are bicharacters of $\mathfrak{p}_{A}$ which are $\subseteq$ invariant; the previous lemma implies that:

$$
\begin{aligned}
& b\left(X, Y ; X^{\prime}, Y^{\prime}\right) \\
& =(-1)^{\alpha_{1}\left|X \cap X^{\prime}\right|+\beta_{1}|X|\left|X^{\prime}\right|+\alpha_{2}\left|X \cap X^{\prime}\right|+\beta_{2}|X|\left|Y^{\prime}\right|} \\
& \quad(-1)^{\alpha_{3}\left|Y \cap X^{\prime}\right|+\beta_{3}|Y|\left|X^{\prime}\right|+\alpha_{4}\left|Y \cap Y^{\prime}\right|+\beta_{4}|Y|\left|Y^{\prime}\right| .}
\end{aligned}
$$


The symmetry of $b$ implies that $\alpha_{2}=\alpha_{3}, \beta_{2}=\beta_{3}$;

$$
\begin{aligned}
b & \left(X, Y ; X^{\prime}, Y^{\prime}\right) \\
= & (-1)^{\alpha_{1}\left|X \cap X^{\prime}\right|+\beta_{1}|X|\left|X^{\prime}\right|+\alpha_{2}\left(\left|X \cap Y^{\prime}\right|+\left|Y \cap X^{\prime}\right|\right)} \\
& (-1)^{\beta_{2}\left(|X|\left|Y^{\prime}\right|+|Y|\left|X^{\prime}\right|\right)+\alpha_{4}\left|Y \cap Y^{\prime}\right|+\beta_{4}|Y|\left|Y^{\prime}\right|} .
\end{aligned}
$$

The $\tau_{\theta_{0}}$ symmetry implies that $\alpha_{1}=\alpha_{4}, \beta_{1}=\beta_{4}$ :

$$
\begin{aligned}
b & \left(X, Y ; X^{\prime}, Y^{\prime}\right) \\
= & (-1)^{\alpha_{1}\left(\left|X \cap X^{\prime}\right|+\left|Y \cap Y^{\prime}\right|\right)+\beta_{1}\left(|X|\left|X^{\prime}\right|+|Y|\left|Y^{\prime}\right|\right)} \\
& (-1)^{\alpha_{2}\left(\left|X \cap Y^{\prime}\right|+\left|X^{\prime} \cap Y\right|\right)+\beta_{2}\left(|X|\left|Y^{\prime}\right|+\left|X^{\prime}\right||Y|\right) .} .
\end{aligned}
$$

Lemma (2.27). Let $b$ a bicharacter of $\mathfrak{p}_{\Lambda} \times p_{\Lambda}$ as in Lemma (2.26), if $\alpha_{1}=\alpha_{2}$, then it is degenerate.

Indeed

$$
\begin{aligned}
& b\left(X, Y ; X^{\prime}, Y^{\prime}\right) \\
& =(-1)^{\alpha_{1}\left(\left|X \cap X^{\prime}\right|+\left|X \cap Y^{\prime}\right|+\left|X^{\prime} \cap Y\right|+\left|Y \cap Y^{\prime}\right|\right)}(-1)^{\beta_{1}\left(|X|\left|X^{\prime}\right|+|Y|\left|Y^{\prime}\right|\right)+\beta_{2}\left(|X|\left|Y^{\prime}\right|+\left|X^{\prime}\right||Y|\right)} .
\end{aligned}
$$

If $X=Y$ and $|X|=2 K$ for some integer $K \neq 0$

$$
b\left(X, Y ; X^{\prime}, Y^{\prime}\right)=1, \quad \forall X^{\prime}, Y^{\prime} \in \mathfrak{p}_{\Lambda} .
$$

Lemma (2.28). The following four bicharacters do not fulfil the condition v):

$$
\begin{aligned}
& (-1)^{\left|X \cap X^{\prime}\right|+\left|Y \cap Y^{\prime}\right|} \\
& (-1)^{\left|X \cap X^{\prime}\right|+\left|Y \cap Y^{\prime}\right|+|X|\left|Y^{\prime}\right|+\left|X^{\prime}\right||Y|} \\
& (-1)^{\left|X \cap Y^{\prime}\right|+\left|X^{\prime} \cap Y\right|+|X|\left|X^{\prime}\right|+|Y|\left|Y^{\prime}\right|} \\
& (-1)^{\left|X \cap Y^{\prime}\right|+\left|X^{\prime} \cap Y\right|+(|X|+|Y|)\left(\left|X^{\prime}\right|+\left|Y^{\prime}\right|\right) .}
\end{aligned}
$$

Indeed for $(X, Y)=\left(X^{\prime}, Y^{\prime}\right)$ they are respectively

$$
\begin{aligned}
& (-1)^{|X|+|Y|} \\
& (-1)^{|X|+|Y|} \\
& (-1)^{|X|^{2}+|Y|^{2}} \\
& (-1)^{(|X|+|Y|)^{2}} .
\end{aligned}
$$

They all are equal to -1 if $Y=\emptyset$ and $|X|=2 K+1$ for instance.

Hence we are left with the four possibilities of the proposition. Nevertheless we shall prove the following lemma.

\section{Lemma (2.29).}

$$
\begin{aligned}
& b^{\sigma}\left(X, Y ; X^{\prime}, Y^{\prime}\right)=(-1)^{\left|X \cap Y^{\prime}\right|+\left|X^{\prime} \cap Y\right|+|X|\left|Y^{\prime}\right|+\left|X^{\prime}\right||Y|} \\
& b^{\varphi}\left(X, Y ; X^{\prime}, Y^{\prime}\right)=(-1)^{\left|X \cap X^{\prime}\right|+\left|Y \cap Y^{\prime}\right|+|X|\left|X^{\prime}\right|+|Y|\left|Y^{\prime}\right|} .
\end{aligned}
$$

are degenerate if and only if $|\Lambda|=2 N+1$. 
Indeed for $X=Y=\Lambda,|\Lambda|=2 N+1$

$$
\begin{aligned}
& b^{\sigma}\left(\Lambda, \Lambda ; X^{\prime}, Y^{\prime}\right)=(-1)^{\left|X^{\prime}\right|+\left|X^{\prime}\right|+\left|X^{\prime}\right|+\left|Y^{\prime}\right|}=1 \\
& b^{\varphi}\left(\Lambda, \Lambda ; X^{\prime}, Y^{\prime}\right)=(-1)^{\left|X^{\prime}\right|+\left|Y^{\prime}\right|+\left|X^{\prime}\right|+\left|Y^{\prime}\right|}=1 .
\end{aligned}
$$

If now $|\Lambda|=2 N$ :

$$
b^{\sigma}\left(\Lambda, \Lambda ; X^{\prime}, Y^{\prime}\right)=b^{\varphi}\left(\Lambda, \Lambda ; X^{\prime}, Y^{\prime}\right)=(-1)^{\left|X^{\prime}\right|+\left|Y^{\prime}\right|} .
$$

On the other hand if

$$
b^{\sigma}\left(X, Y ; X^{\prime}, Y^{\prime}\right)=b^{\varphi}\left(X, Y ; X^{\prime}, Y^{\prime}\right)=1, \quad \forall X^{\prime}, Y^{\prime} \in \mathfrak{p}_{A},
$$

in particular for $Y^{\prime}=\emptyset$ :

$$
(-1)^{\left|Y \cap X^{\prime}\right|+\left|X^{\prime}\right||Y|}=\left((-1)^{\left|X \cap X^{\prime}\right|+|X|\left|X^{\prime}\right|}=\right)=1, \quad \forall X^{\prime} \subset \Lambda,
$$

for $X^{\prime}$ such that $\left|X^{\prime}\right|=1$ and $\left.\mid X^{\prime} \cap Y\right)\left(\right.$ or $\left.\left|X^{\prime} \cap X\right|\right)=0$

$$
(-1)^{|Y|}=\left((-1)^{|X|}=\right)=1 \text {, }
$$

but for $X^{\prime}$ such that $\left|X^{\prime}\right|=1$ and $\left|X^{\prime} \cap Y\right|\left(\right.$ or $\left.\left|X^{\prime} \cap X\right|\right)=1$

$$
(-1)^{|Y|}=\left((-1)^{|X|}=\right)=-1 \text {, }
$$

hence a contradiction; since $Y$ (or $X$ ) are different from $\Lambda$ as we have seen for $|\Lambda|=2 N$ and of course for $|\Lambda|=\infty ; Y$ (or $X$ ) are the void set; by symmetry $X$ (or $Y$ ) are the void set which concludes the proof.

The next lemma shows that $b^{S}$ and $b^{F}$ are non degenerate.

Lemma (2.30). $b^{S}$ and $b^{F}$ are non degenerate.

Indeed if $X, Y \in \mathfrak{p}_{A}$ are such that:

$$
(-1)^{\left|X \cap Y^{\prime}\right|+\left|X^{\prime} \cap Y\right|}=1, \quad \forall X^{\prime}, Y^{\prime} \in \mathfrak{p}_{\Lambda} .
$$

in particular for $Y^{\prime}=\emptyset$ :

$$
(-1)^{\left|X^{\prime} \cap Y\right|}=1, \quad \forall X^{\prime} \in \mathfrak{p}_{A},
$$

which implies that $Y=\emptyset$; symmetrically one can prove that the first relation implies $X=\emptyset$.

On the other hand if

$$
(-1)^{\left|X \dot{n} X^{\prime}\right|+\left|Y \cap Y^{\prime}\right|+(|X|+|Y|)\left(\left|X^{\prime}\right|+\left|Y^{\prime}\right|\right)}=1, \quad \forall X^{\prime}, Y^{\prime} \in \mathfrak{p}_{A},
$$

then in particular for $X^{\prime}=Y^{\prime}$ :

$$
(-1)^{\left|X \triangle Y \cap X^{\prime}\right|}=1, \quad \forall X^{\prime} \in \mathfrak{p}_{A},
$$

which implies that $X=Y$; but then

$$
(-1)^{\left|X \cap X^{\prime} \Delta Y^{\prime}\right|}=1, \quad \forall X^{\prime}, Y^{\prime} \in \mathfrak{p}_{\Lambda},
$$

which in turn implies $X=\emptyset$ hence $Y=\emptyset$. 
Finally $b^{S}, b^{F}, b^{\sigma}, b^{\varphi}$ fulfil the condition $\left.\mathrm{v}\right)$, so

$$
\begin{aligned}
& b^{S}(X, Y ; X, Y)=(-1)^{2|X \cap Y|}=1 \\
& b^{F}(X, Y ; X, Y)=(-1)^{|X|+|Y|+(|X|+|Y|)^{2}}=1 \\
& b^{\varphi}(X, Y ; X, Y)=(-1)^{|X|+|X|^{2}+|Y|+|Y|^{2}}=1 \\
& b^{\sigma}(X, Y ; X, Y)=(-1)^{2|X \cap Y|+2|X||Y|}=1 .
\end{aligned}
$$

The next propositions shows the relations between these bicharacters.

Proposition (2.31). Let $\theta_{i}$ be the homomorphisms of $\mathfrak{p}_{A}$ defined in (2.14); then we have the following relations

$$
\begin{aligned}
& b^{F}\left(X, Y ; X^{\prime}, Y^{\prime}\right)=b^{S}\left(\tau_{\theta_{1}}(X, Y), \tau_{\theta_{1}}\left(X^{\prime}, Y^{\prime}\right)\right) . \\
& b^{\varphi}\left(X, Y ; X^{\prime}, Y^{\prime}\right)=b^{\sigma}\left(\tau_{\theta_{2}}(X, Y), \tau_{\theta_{2}}\left(X^{\prime}, Y^{\prime}\right)\right) .
\end{aligned}
$$

The proof of this proposition is not difficult but tedious, see Appendix A.

At this stage it is worth-while to show that these four bicharacters correspond to the actual extensions of $\mathfrak{p}_{\boldsymbol{A}} \times \mathfrak{p}_{\boldsymbol{A}}$. Let us consider the commutation relations of the generators:

a) for $b^{S}$

$$
\begin{aligned}
& b^{S}(\{i\}, \emptyset ;\{j\}, \emptyset)=b^{S}(\emptyset ;\{i\} ; \emptyset,\{j\})=1 \\
& b^{S}(\{i\}, \emptyset ; \emptyset,\{j\})=b^{S}(\emptyset,\{i\} ;\{j\}, \emptyset)=1-2 \delta_{i j} .
\end{aligned}
$$

these are the commutation relations of a quantum spin system.

b) for $b^{F}$

$$
\begin{aligned}
& b^{F}(\{i\}, \emptyset ; \emptyset,\{j\})=b^{F}(\emptyset,\{i\} ;\{j\}, \emptyset)=-1, \\
& b^{F}(\{i\}, \emptyset ;\{j\}, \emptyset)=b^{F}(\emptyset,\{i\} ; \emptyset,\{j\})=2 \delta_{i j}-1 .
\end{aligned}
$$

these are the commutation relations of a Clifford algebra.

c) for $b^{\varphi}$

$$
\begin{aligned}
& b^{\varphi}(\{i\}, \emptyset ; \emptyset,\{j\})=b^{\varphi}(\emptyset,\{\mathrm{i}\} ;\{j\}, \emptyset)=1, \\
& b^{\varphi}(\{i\}, \emptyset ;\{j\}, \emptyset)=b^{\varphi}(\emptyset,\{i\} ; \emptyset,\{j\})=2 \delta_{i j}-1 .
\end{aligned}
$$

so that the systems splits into the tensor product of two Clifford algebras.

d) for $b^{\sigma}$

$$
\begin{aligned}
& b^{\sigma}(\{i\}, \emptyset ;\{j\}, \emptyset)=b^{\sigma}(\emptyset,\{i\} ; \emptyset,\{j\})=1, \\
& b^{\sigma}(\{i\}, \emptyset ; \emptyset,\{j\})=b^{\sigma}(\emptyset,\{i\} ;\{j\}, \emptyset)=2 \delta_{i j}-1
\end{aligned}
$$

the system is of quantum spin type.

We describe in the next proposition the multipliers whose associated bicharacters are respectively $b^{S}, b^{F}, b^{\varphi}$ and $b^{\sigma}$. They are chosen such that $\xi(e, e)$ $=\xi\left(g, g^{-1}\right)=1$; in such a way the generators of $\Delta(G, \xi)$ are both unitary and hermitian $^{1}$.

1 These conditions are not sufficient to determine completely the $\xi$ since if $\mu$ is a function from $\mathfrak{p}_{\Lambda} \times \mathfrak{p}_{\Lambda}$ into \pm 1 which is 1 on $(\emptyset, \emptyset)$. Then:

$\xi^{\prime}\left(X, Y ; X^{\prime}, Y^{\prime}\right)=\mu(X, Y) \mu\left(X^{\prime}, Y^{\prime}\right) \mu\left(X \triangle X^{\prime}, Y \triangle Y^{\prime}\right) \xi\left(X, Y ; X^{\prime}, Y^{\prime}\right)$,

satisfy the same conditions 
Proposition (2.32). The following multipliers are associated respectively to $b^{S}, b^{F}$, $b^{\varphi}$ and $b^{\sigma}$ :

$$
\begin{aligned}
& \xi^{S}\left(X, Y ; X^{\prime}, Y^{\prime}\right)=i^{-|X \cap Y|} i^{-\left|X^{\prime} \cap Y^{\prime}\right|} i^{\left|X \Delta X^{\prime} \cap Y \Delta Y^{\prime}\right|}(-1)^{\left|X^{\prime} \cap Y\right|}, \quad \forall X, X^{\prime}, Y, Y^{\prime} \in \mathfrak{p}_{A} . \\
& \xi^{F}\left(X, Y ; X^{\prime}, Y^{\prime}\right)=i^{\left|X \cap \theta_{1}(X)\right|+\left|Y \cap \theta_{1}(Y)\right|+|X||Y|} \\
& i^{\left|X^{\prime} \cap \theta_{1}\left(X^{\prime}\right)\right|+\left|Y^{\prime} \cap \theta_{1}\left(Y^{\prime}\right)\right|+\left|X^{\prime}\right|\left|Y^{\prime}\right|-\left|X \Delta X^{\prime} \cap \theta_{1}\left(X \Delta X^{\prime}\right)\right|} \\
& i^{-\left|Y \Delta Y^{\prime} \cap \theta_{1}\left(Y \Delta Y^{\prime}\right)\right|-\left|X \Delta X^{\prime}\right|\left|Y \Delta Y^{\prime}\right|}(-1)^{\left|X^{\prime}\right||Y|+\left|X^{\prime} \cap \theta_{1}(X)\right|+\left|Y^{\prime} \cap \theta_{1}(Y)\right|}, \\
& \xi^{\varphi}\left(X, Y ; X^{\prime}, Y^{\prime}\right)=i^{\left|X \cap \theta_{2}(X)\right|+\left|Y \cap \theta_{2}(Y)\right|} \\
& i^{\left|X^{\prime} \cap \theta_{2}\left(X^{\prime}\right)\right|+\left|Y^{\prime} \cap \theta_{2}\left(Y^{\prime}\right)\right|-\left|X \Delta X^{\prime} \cap \theta_{2}\left(X \Delta X^{\prime}\right)\right|-\left|Y \Delta Y^{\prime} \cap \theta_{2}\left(Y \Delta Y^{\prime}\right)\right|}(-1)^{\left|X^{\prime} \cap \theta_{2}(X)\right|+\left|Y^{\prime} \cap \theta_{2}(Y)\right|}, \\
& \xi^{\sigma}\left(X, Y ; X^{\prime}, Y^{\prime}\right)=i^{-|X||Y|-|X \cap Y|} i^{-\left|X^{\prime}\right|\left|Y^{\prime}\right|-\left|X^{\prime} \cap Y^{\prime}\right|} i^{\left|X \Delta X^{\prime}\right|\left|Y \Delta Y^{\prime}\right|+\left|X \Delta X^{\prime} \cap Y \Delta Y^{\prime}\right|} \\
& (-1)^{\left|X^{\prime}\right||Y|+\left|X^{\prime} \cap Y\right|}, \quad \forall X, Y, X^{\prime}, Y^{\prime} \in \mathfrak{P}_{A} .
\end{aligned}
$$

They are chosen to satisfy:

$$
\xi(X, Y ; X, Y)=1, \quad \forall X, Y \in \mathfrak{p}_{A} .
$$

From Proposition (2.31) one can conclude that

i) $\xi^{F}\left(X, Y ; X^{\prime}, Y^{\prime}\right)=F_{\theta_{1}}(X, Y) F_{\theta_{1}}\left(X^{\prime}, Y^{\prime}\right) F_{\theta_{1}}\left(X \triangle X^{\prime}, Y \triangle Y^{\prime}\right)$

$$
\cdot \xi^{S}\left(\tau_{\theta_{1}}(X, Y) ; \tau_{\theta_{1}}\left(X^{\prime}, Y^{\prime}\right)\right), \quad \forall X, Y, X^{\prime}, Y^{\prime} \in \mathfrak{p}_{\Lambda} .
$$

ii) $\xi^{\varphi}\left(X, Y ; X^{\prime}, Y^{\prime}\right)=G_{\theta_{2}}(X, Y) G_{\theta_{2}}\left(X^{\prime}, Y^{\prime}\right) G_{\theta_{2}}\left(X \triangle X^{\prime}, Y \triangle Y^{\prime}\right)$

$$
\cdot \xi^{\sigma}\left(\tau_{\theta_{2}}(X, Y) ; \tau_{\theta_{2}}\left(X^{\prime}, Y^{\prime}\right)\right), \quad \forall X, Y, X^{\prime}, Y^{\prime} \in \mathfrak{P}_{\Lambda} \text {. }
$$

where

$$
\begin{aligned}
& F_{\theta_{1}}(X, Y)=(-1)^{\left|Y \cap \theta_{1}(Y)\right|+\left|X \cap Y \cap \theta_{1}(X \Delta Y)\right|}(-1)^{|X||Y| \mid}\left(\frac{|X|+|Y|}{2}\right)+|X||Y|+|X \cap Y| \\
& \forall X, Y \in \mathfrak{P}_{A} . \\
& G_{\theta_{2}}(X, Y)=F_{\theta_{1}}(X, Y) i^{\left|\theta_{2}(X \Delta Y)\right|(|X|+|Y|)}(-1)^{\left|\theta_{2}(X) \cap \theta_{2}(Y)\right|} \\
& \cdot(-1)^{\left|\theta_{1}(Y)\right|}(-1)^{\left|\theta_{1}(X) \cap \theta_{1}(Y)\right|}(-1)^{\left|X \cap \theta_{2}(X \Delta Y)\right||Y|+\left|Y \cap \theta_{2}(X \triangle Y)\right||X|} .
\end{aligned}
$$

moreover $F_{\theta_{1}}(X, Y)$ and $G_{\theta_{2}}(X, Y)$ are equal to \pm 1 and $F_{\theta_{1}}(\emptyset, \emptyset)=G_{\theta_{1}}(\emptyset, \emptyset)=1$. They can computed by a tendious calculation using essentially Proposition (2.15).

From this result and using Proposition (1.16) we can conclude the following theorem which is nothing but the fact the Clifford algebra is isomorphic to the U.H.F. algebra.

Theorem (2.36). Let $\xi^{S}$, $\xi^{F}$ (resp. $\left.\xi^{\sigma}, \xi^{\varphi}\right)$ be the multipliers of $p_{\Lambda} \times p_{A}$ previously defined. The algebras $\overline{\Delta\left(\mathfrak{p}_{\Lambda} \times \mathfrak{p}_{\Lambda}, \xi^{S}\right)}$ and $\overline{\Delta\left(\mathfrak{p}_{\Lambda} \times \mathfrak{p}_{\Lambda}, \xi^{F}\right)}$ (resp. $\overline{\Delta\left(\mathfrak{p}_{\Lambda} \times \mathfrak{p}_{\Lambda}, \xi^{\varphi}\right)}$ and

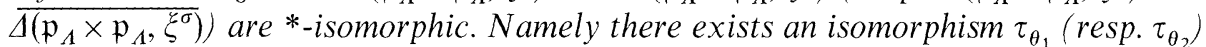
of $\mathfrak{p}_{A} \times \mathfrak{p}_{A}$ and a function $F_{\theta_{1}}\left(\right.$ resp. $G_{\theta_{2}}$ ) from $\mathfrak{p}_{A} \times \mathfrak{p}_{A}$ to \pm 1 such that $\hat{\alpha}_{1}$ (resp. $\hat{\alpha}_{2}$ ) defined by:

$$
\begin{aligned}
\hat{\alpha}_{1}\left(\delta_{X, Y}^{F}\right) & =F_{\theta_{1}}(X, Y) \delta_{\tau_{\theta_{2}}}^{S}(X, Y) \\
\left(\text { resp. } \hat{\alpha}_{2}\left(\delta_{X, Y}^{\varphi}\right)\right. & \left.=G_{\theta_{2}}(X, Y) \delta_{\tau_{\theta_{2}}}^{\sigma}(X, Y)\right) . \quad \forall X, Y \in \mathfrak{p}_{\Lambda},
\end{aligned}
$$


realizes this isomorphism which is a symmetry, i.e. it satisfies $\left(\hat{\alpha}_{1}\right)^{2}=i$, resp. $\left.\left(\hat{\alpha}_{2}\right)^{2}=i\right)$. $\theta_{1}$ (resp. $\theta_{2}$ ) is given by (2.14) and $F_{\theta_{1}}$ (resp. $G_{\theta_{2}}$ ) is given by (2.34) (resp. (2.35)).

It is clear if one restricts to the sets $(\{i\}, \emptyset)$ and $(\emptyset,\{j\})$ that these isomorphisms are the abstract version of the usual trick which allows to transform the generators of the U.H.F. algebra into the generators of the Clifford algebra (see e.g. [4]).

For the sake of completeness we shall derive a result which shows that $b^{\varphi}$ and $b^{F}$ are connected in the previous way if $|\Lambda|$ is even or infinite; if $|\Lambda|$ is odd the degeneracy of $b^{\varphi}$ excludes this fact.

Proposition (2.37). If $|\Lambda|$ is even or infinite there exist isomorphisms $\tau_{21}$ of $\mathfrak{p}_{\Lambda} \times \mathfrak{p}_{\Lambda}$ such that

$$
b^{1}\left(X, Y ; X^{\prime}, Y^{\prime}\right)=b^{2}\left(\tau_{21}(X, Y), \tau_{21}\left(X^{\prime}, Y^{\prime}\right)\right), \quad \forall X, Y, X^{\prime}, Y^{\prime} \in \mathfrak{p}_{\Lambda},
$$

where $b^{1}$ and $b^{2}$ are any of the two bicharacters described in Proposition (2.24).

Indeed it has been proved that $b^{F}$ and $b^{S}$ (resp. $b^{\varphi}$ and $b^{\sigma}$ ) are connected by such an isomorphism, hence it is sufficient to prove the statement for $b^{S}$ and $b^{\varphi}$ for instance.

It can be shown that if it exists it cannot be of the form (2.8); namely it cannot commute with $\tau_{\theta_{0}}$. We can give an abstract form of this isomorphism; nevertheless it is perhaps more instructive to give an expression which connects the familiar generators; let $\left(\sigma_{i}^{x}, \sigma_{i}^{y}\right)_{i \in N}$ be the generators of the usual quantum spin system; define

$$
\begin{aligned}
& b\left(e_{2 i+1}\right)=\prod_{j<i} \sigma_{2 j+1}^{x} \sigma_{2 j+1}^{y} \sigma_{2 i+1}^{x}, \\
& b\left(e_{2 i+2}\right)=\prod_{j<i} \sigma_{2 j+1}^{x} \sigma_{2 j+1}^{y} \sigma_{2 i+1}^{y}, \\
& b\left(f_{2 i+1}\right)=\prod_{j<i} \sigma_{2 j+2}^{x} \sigma_{2 j+2}^{y} \sigma_{2 i+2}^{x}, \\
& b\left(f_{2 i+2}\right)=\prod_{j<i} \sigma_{2 j+2}^{x} \sigma_{2 j+2}^{y} \sigma_{2 i+2}^{y} .
\end{aligned}
$$

It can be shown very easily that the $b\left(e_{i}\right)$ 's and the $b\left(f_{j}\right)$ 's are the generators of $\overline{\Delta\left(\mathfrak{p}_{A} \times \mathfrak{p}_{\Lambda}, \xi^{\varphi}\right)}$. From this expression one can induce the form of the isomorphism on the generating elements of $\mathfrak{p}_{A} \times \mathfrak{p}_{A}$ :

$$
\begin{aligned}
\tau_{S \varphi}(\{1\}, \emptyset) & =(\{1\}, \emptyset), \\
\tau_{S \varphi}(\{2 i+1\}, \emptyset) & =(\{1,3, \ldots, 2 i+1\},\{1,3, \ldots, 2 i-1\}), \quad i>0, \\
\tau_{S \varphi}(\{2\}, \emptyset) & =(\emptyset,\{1\}), \\
\tau_{S \varphi}(\{2 i+2\}, \emptyset) & =(\{1,3, \ldots, 2 i-1\},\{1,3, \ldots, 2 i+1\}), \quad i>0, \\
\tau_{S \varphi}(\emptyset,\{1\}) & =(\{2\}, \emptyset), \\
\tau_{S \varphi}(\emptyset,\{2 i+1\}) & =(\{2,4, \ldots, 2 i+2\},\{2,4, \ldots, 2 i\}), \quad i>0, \\
\tau_{S \varphi}(\emptyset,\{2\}) & =(\emptyset,\{2\}), \\
\tau_{S \varphi}(\emptyset,\{2 i+2\}) & =(\{2,4, \ldots, 2 i\},\{2,4, \ldots, 2 i+2\}), \quad i>0 .
\end{aligned}
$$

This form shows that $|\Lambda|$ has to be even. It is not unique but it will be useful for the proof of Corollary (2.41). 
For the sake of completeness, in Appendix B we give explicit formulas for the other isomorphisms which connect the different bicharacters.

Theorem (2.40). Any two of the algebras $\overline{\Delta\left(p_{A} \times p_{A}, \xi\right)}$, where $\xi$ is a multiplier satisfying the conditions of Proposition (2.32), are *-isomorphic; the isomorphism $\hat{\alpha}_{21}$ can be chosen such that:

$$
\hat{\alpha}_{21}\left(\delta_{x, Y}^{1}\right)=f_{21}(X, Y) \delta_{\tau_{21}(X, Y)}^{2}, \quad \forall X, Y \in \mathfrak{p}_{A},
$$

where $f_{21}$ is a function from $\mathfrak{p}_{\Lambda} \times \mathfrak{p}_{\Lambda} \rightarrow\{ \pm 1\}$ and $\tau_{21}$ is an isomorphism of $\mathfrak{p}_{\Lambda} \times \mathfrak{p}_{\Lambda}$ $\rightarrow \mathfrak{p}_{A} \times p_{A}$ of the form:

$$
\tau_{21}(X ; Y)=\left(X \triangle F_{(21) 1}(X) \triangle F_{(21) 2}(Y) ; Y \triangle G_{(21) 1}(Y) \triangle G_{(21) 2}(X)\right)
$$

where the $F_{(21) i}$ 's and the $G_{(21) i}$ 's are homomorphisms of $\mathfrak{p}_{\Lambda}$.

Proof. The proof is a simple application of the previous results and essentially Proposition (2.7).

For the sake of completeness we shall add a result pertinent to the Clifford algebra.

Corollary (2.41). Let $\mathscr{H}_{1}$ and $\mathscr{H}_{2}$ be real Hilbert space of even or infinite dimensions; $\mathscr{H}=\mathscr{H}_{1} \oplus \mathscr{H}_{2}$ their orthogonal sum. Let $\mathfrak{U}(\mathscr{H})$ (resp. $\left.\mathfrak{\mathfrak { U }}\left(\mathscr{H}_{i}\right)\right)$ be the Clifford algebra built on $\mathscr{H}\left(\right.$ resp. $\left.\mathscr{H}_{i}\right)$; then $\mathfrak{U}(\mathscr{H})$ is $*$-isomorphic to $\mathfrak{A}\left(\mathscr{H}_{1}\right) \otimes \mathfrak{U}\left(\mathscr{H}_{2}\right)$. The isomorphism can be chosen such that it sends a finite product of generators in $\mathfrak{U}\left(\mathscr{H}_{1}\right) \otimes \mathfrak{A}\left(\mathscr{H}_{2}\right)$ into a finite product of generators in $\mathfrak{A}(\mathscr{H})$.

Proof. If $\operatorname{dim} \mathscr{H}_{1}=\operatorname{dim} \mathscr{H}_{2}$ it is a direct application of formulas (2.38)

$$
\mathfrak{U}\left(\mathscr{H}_{1}\right) \otimes \mathfrak{H}\left(\mathscr{H}_{2}\right) \simeq \overline{\Delta\left(\mathfrak{p}_{A} \times \mathfrak{p}_{A} ; \xi^{\varphi}\right)}
$$

for a set $\Lambda$ with $|\Lambda|=\operatorname{dim} \mathscr{H}_{i}$; and

$$
\mathfrak{A}(\mathscr{H}) \simeq \overline{\Delta\left(\mathfrak{p}_{A} \times \mathfrak{p}_{A} ; \xi^{F}\right)}
$$

For $\operatorname{dim} \mathscr{H}_{1} \neq \operatorname{dim} \mathscr{H}_{2}$ one can built explicitly the isomorphism using formulas similar to the formulas (2.38).

\section{Appendix A}

Let us prove the relation

$$
b^{F}\left(X, Y ; X^{\prime}, Y^{\prime}\right)=b^{S}\left(\tau_{\theta_{1}}(X, Y) ; \tau_{\theta_{1}}\left(X^{\prime}, Y^{\prime}\right)\right) .
$$

One has:

(A.1) $b^{S}\left(\tau_{\theta_{1}}(X, Y) ; \tau_{\theta_{1}}\left(X^{\prime}, Y^{\prime}\right)\right)$

$$
=(-1)^{\left|X \Delta \theta_{1}(X \Delta Y) \cap Y^{\prime} \Delta \theta_{1}\left(X^{\prime} \Delta Y^{\prime}\right)\right|}(-1)^{\left|X^{\prime} \Delta \theta_{1}\left(X^{\prime} \Delta Y^{\prime}\right) \cap Y \Delta \theta_{1}(X \Delta Y)\right|} \text {. }
$$

Since $X, Y \rightarrow(-1)^{|X \cap Y|}$ is a bicharacter of $p_{A}$ :

(A.2) $\quad b^{S}\left(\tau_{\theta_{1}}(X, Y) ; \tau_{\theta_{1}}\left(X^{\prime}, Y^{\prime}\right)\right)$ $=(-1)^{\left|X \cap Y^{\prime}\right|+\left|X^{\prime} \cap Y\right|+\left|X \Delta Y \cap \theta_{1}\left(X^{\prime} \Delta Y^{\prime}\right)\right|+\left|X^{\prime} \Delta Y^{\prime} \cap \theta_{1}(X \Delta Y)\right|}$. 
Then using the formula (2.16)

(A.3) $\quad b^{S}\left(\tau_{\theta_{1}}(X, Y) ; \tau_{\theta_{1}}\left(X^{\prime}, Y^{\prime}\right)\right)$

$$
=(-1)^{\left|X \cap Y^{\prime}\right|+\left|X^{\prime} \cap Y\right|+|X \Delta Y|\left|X^{\prime} \Delta Y^{\prime}\right|+\left|X \Delta Y \cap X^{\prime} \Delta Y^{\prime}\right|}
$$

Using again the fact that $X, Y \rightarrow(-1)^{|X \cap Y|}$ is a bicharacter of $\mathfrak{p}_{A}$ and the obvious formula

(A.4) $|X \triangle Y|=|X|+|Y|-2|X \cap Y|$

(A.5) $\quad b^{S}\left(\tau_{\theta_{1}}(X, Y) ; \tau_{\theta_{1}}\left(X^{\prime}, Y^{\prime}\right)\right)=(-1)^{\left|X \cap X^{\prime}\right|+\left|Y \cap X^{\prime}\right|+(|X|+|Y|)\left(\left|X^{\prime}\right|+\left|Y^{\prime}\right|\right)}$.

In order to prove that

$$
b^{\varphi}\left((X, Y) ;\left(X^{\prime}, Y^{\prime}\right)\right)=b^{\sigma}\left(\tau_{\theta_{2}}(X, Y) ; \tau_{\theta_{2}}\left(X^{\prime}, Y^{\prime}\right)\right)
$$

one proves first that

$$
\begin{aligned}
\text { (A.6) } & (-1)^{\left|X \Delta \theta_{2}(X \Delta Y) \cap Y^{\prime} \Delta \theta_{2}\left(X^{\prime} \Delta Y^{\prime}\right)\right|}(-1)^{\left|X^{\prime} \Delta \theta_{2}\left(X^{\prime} \Delta Y^{\prime}\right) \cap Y \Delta \theta_{2}(X \Delta Y)\right|} \\
= & (-1)^{\left|X \cap X^{\prime}\right|+\left|Y \cap Y^{\prime}\right|+(|X|+|Y|)\left(\left|X^{\prime}\right|+\left|Y^{\prime}\right|\right)}
\end{aligned}
$$

in the same way as for $\theta_{1}$ since $\theta_{2}$ satisfies (2.19) which is the same than (2.16).

Moreover

(A.7) $\quad(-1)^{\left|X \cap \theta_{2}(X \Delta Y)\right|\left|Y^{\prime} \Delta \theta_{2}\left(X^{\prime} \Delta Y^{\prime}\right)\right|}(-1)^{\left|X^{\prime} \cap \theta_{2}\left(X^{\prime} \Delta Y^{\prime}\right)\right|\left|Y \Delta \theta_{2}(X \Delta Y)\right|}=(-1)^{|X|\left|Y^{\prime}\right|+\left|X^{\prime}\right||Y|}$

if one uses (A.4) and the relation (2.18). Combining (A.6) and (A.7), one proves (2.31).

\section{Appendix B}

In this appendix, we give explicit formulas for the different isomorphisms $\tau_{i j}$ which connect different multipliers.

Let us remark that since one has

$$
\begin{array}{ll}
\text { (B.1) } \quad \tau_{i j}=\tau_{j i}^{-1} \\
\\
\tau_{i j} \tau_{j k}=\tau_{i k}
\end{array}
$$

they are all obtained by suitable products of $\tau_{\theta_{1}}, \tau_{\theta_{2}}, \tau_{\varphi S}$ and $\tau_{S \varphi}$. Moreover we use the following compact notations

(B.2) $N_{k}=\left\{x_{i} ; i \leqq k\right\} \quad k>0$

(B.3) $E_{k}=\left\{x_{2 i} ; i \leqq k\right\} \quad k>0$

(B.4) $O_{k}=\left\{x_{2 i-1} ; i \leqq k\right\} \quad k>0$

(B.5) $N_{0}=E_{0}=O_{0}=\emptyset$.

Table 1 gives the ten different $\tau_{i j}$. Notice that $\tau_{\theta_{1}}^{2}=\tau_{\theta_{2}}^{2}=i$. 
Table 1. Different isomorphisms of Theorem (2.37)

\begin{tabular}{|c|c|c|c|}
\hline$(\{2 i+1\}, \emptyset)$ & $(\{2 i+2\}, \emptyset)$ & $(\emptyset,\{2 i+1\})$ & $(\emptyset,\{2 i+2\})$ \\
\hline$\tau_{\theta_{1}}\left(N_{2 \imath+1}, N_{2 i}\right)$ & $\left(N_{2 \imath+2}, N_{2 \imath+1}\right)$ & $\left(N_{2 l}, N_{2 l+1}\right)$ & $\left(N_{2 t+1}, N_{2 t+2}\right)$ \\
\hline$\tau_{\theta_{2}}\left(N_{2 i+1}, N_{2 i}\right)$ & $\left(N_{2 \imath+1}, N_{2 \imath+2}\right)$ & $\left(N_{2 i}, N_{2 i+1}\right)$ & $\left(N_{2 i+2}, N_{2 i+1}\right)$ \\
\hline$\tau_{S \varphi}\left(O_{i+1}, O_{\imath}\right)$ & $\left(O_{\imath}, O_{\imath+1}\right)$ & $\left(E_{\imath+1}, E_{i}\right)$ & $\left(E_{i}, E_{i+1}\right)$ \\
\hline$\tau_{\varphi S}\left(N_{2 i+1}, \emptyset\right)$ & $\left(\emptyset, N_{2 \imath+1}\right)$ & $\left(N_{2 i+2} \triangle\{2 i+1\}, \emptyset\right)$ & $\left(\emptyset, N_{2 i+2} \triangle\{2 i+1\}\right)$ \\
\hline$\tau_{F \varphi}\left(E_{i} \triangle\{2 i+1\}, E_{\imath}\right)$ & $\left(E_{l}, E_{\imath} \triangle\{2 i+1\}\right)$ & $\left(O_{i+1} \triangle\{2 i+2\}, O_{1+i}\right)$ & $\left(O_{i+1}, O_{i+1} \triangle\{2 i+2\}\right)$ \\
\hline$\tau_{\varphi F}\left(\{2 i+1\}, N_{2 \ell}\right)$ & $\left(N_{2 i+2},\{2 i+1\}\right)$ & $\left(\{2 i+2\}, N_{2 \imath}\right)$ & $\left(N_{2 i+2},\{2 i+2\}\right)$ \\
\hline$\overline{\tau_{S \sigma}}\left(E_{t} \triangle\{2 i+1\}, E_{i}\right)$ & $\left(E_{i+1} \triangle\{2 i+1\}, E_{i+1}\right)$ & $\left(O_{\imath} \triangle\{2 i+2\}, O_{\imath}\right)$ & $\left(O_{\imath+1} \triangle\{2 i+2\}, O_{t+1}\right)$ \\
\hline$\tau_{\sigma S}\left(N_{2 \imath+1}, \emptyset\right)$ & $\left(\emptyset, N_{2 \imath+1}\right)$ & $\left(N_{2 i+1},\{2 i+1,2 i+2\}\right)$ & $\left(\{2 i+1,2 i+2\}, N_{2 l+1}\right)$ \\
\hline$\overline{\tau_{F \sigma}\left(O_{t+1}, O_{t}\right)}$ & $\left(O_{i+1} \triangle\{2 i+2\}, O_{i} \triangle\{2 i+2\}\right)$ & $\left(E_{\imath+1} \triangle\{2 i+1\}, E_{l} \triangle\{2 i+1\right.$ & $\left(E_{\imath+1}, E_{\imath}\right)$ \\
\hline$\overline{\tau_{\sigma F}}\left(\{2 i+1\}, N_{2 i}\right)$ & $\left(N_{2 l},\{2 i+2\}\right)$ & $\left(\{2 i+1\}, N_{2 i+2}\right)$ & $\left(N_{2 t+2},\{2 i+2\}\right)$ \\
\hline
\end{tabular}

\section{References}

1. Mackey, G.W.: Acta Math. 99, 265 (1958)

2. Sirugue-Collin, M., Sirugue, M.: Commun. math. Phys. 48, 131 (1976)

3. Schultz, T.D., Mattis, D.C., Lieb,E.H.: Rev. Mod. Phys. 36, 856 (1964)

4. Guichardet, A.: Ann. Ecole Norm. Super. 83, 1 (1966)

5. Kleppner, A.: Math. Ann. 158, 11 (1965)

6. Manuceau, J., Sirugue, M., Testard,D., Verbeure, A.: Commun. math. Phys. 32, 231 (1973)

Communicated by H. Araki

Received March 3, 1978; in revised form June 8, 1978 
